\title{
AVALIANDO PARAMETRIZAÇÕES DA CAMADA LIMITE PLANETÁRIA DO WRF UTILIZANDO DADOS DE VELOCIDADE E DIREÇÃO DO VENTO DO PROJETO CHUVA EM FORTALEZA
}

\author{
João Bosco V. Leal Junior ${ }^{1} *$, Cláudio C. Pellegrini ${ }^{2}$, Gerson P. Almeida ${ }^{1}$, Gilberto Fisch ${ }^{3}$, \\ Roberto F. F. Lyra ${ }^{4}$, Rosiberto Salustiano Da Silva Junior ${ }^{4}$.
}

${ }^{1}$ Univ. Estadual do Ceará (UECE); ${ }^{2}$ Univ. Federal de São João Del-Rei (UFSJ); ${ }^{3}$ Instituto de Aeronáutica e Espaço (IAE); ${ }^{4}$ Univ. Federal de Alagoas (UFAL).

\section{*bosco.leal@uece.br.}

RESUMO: O objetivo deste trabalho é avaliar seis esquemas físicos de Camada Limite Planetária (CLP) presentes no modelo WRF v3.3, comparando os resultados de simulações para a velocidade e direção do vento com dados de radiossondagens realizadas em Fortaleza, durante a campanha do Projeto Chuva, em abril de 2011. Todos os esquemas apresentaram índices estatísticos próximos entre si, mostrando que foram capazes de reproduzir, de forma satisfatória, o perfil do vento nos primeiros $5 \mathrm{~km}$ da atmosfera. Os resultados indicam ainda que o modelo apresentou uma tendência de superestimar em média 23,5\% a velocidade do vento, principalmente em condições que houve precipitação.

ABSTRACT: The aim of this work is to evaluate six planetary boundary layer (PBL) schemes available in the WRF v3.3 model, comparing the simulation results for the wind speed and direction against radiosonde data obtained during the Chuva Project campaign at Fortaleza (Brazil) on April 2011. All schemes presented statistical indexes close to each other, showing that they were able to reproduce reasonably the wind profile in the first $5 \mathrm{~km}$ of the atmosphere. The results also indicate that the model showed a tendency to overestimate in average $23.5 \%$ the wind speed, especially for conditions with precipitation.

\section{INTRODUÇÃO}

Durante o mês de abril de 2011, ocorreu em Fortaleza a segunda campanha do projeto CHUVA, que tem como um dos objetivos melhorar o entendimento dos sistemas 
precipitantes sobre diversas regiões do Brasil, principalmente os relacionados a nuvens quentes. O projeto CHUVA proporciona um banco de dados bastante rico para trabalhos de validação e otimização destes modelos. Neste sentido, este trabalho avalia seis esquemas físicos de Camada Limite Planetária (CLP) no modelo WRF, para verificar qual deles melhor descreve a evolução da velocidade e direção do vento, comparando dados de simulação com observações de radiossondagem obtidas durante o experimento em Fortaleza.

\section{MATERIAL E MÉTODOS}

O período de estudo foi de 09 a 12 de abril de 2011, escolhido por ter sido o mais chuvoso do experimento. Foram utilizadas 15 radiossondagens, todas lançadas da estação meteorológica do Inmet em Fortaleza nos horários de 00, 06, 12 e 18 UTC.

O modelo WRF (núcleo ARW v.3.3) foi configurado com três grades aninhadas, concêntricas na estação do Inmet em Fortaleza. O domínio do modelo na horizontal tinha grades com resoluções de 90, 18 e 3,6 km com 30x30, 36x36 e 61x61 pontos, respectivamente. Na vertical, foram utilizados 45 níveis, com o topo do modelo no nível de $100 \mathrm{hPa}$, correspondendo em média a cerca de $16 \mathrm{~km}$ de altura. As condições iniciais e de contorno foram obtidas a partir das análises FNL do NCEP.

Os esquemas físicos adotados foram os mais usuais encontrados na literatura, como o esquema RRTM para a radiação de onda longa, Dudhia para a radiação de onda curta, WSM6 para a microfísica, Kain-Fritsch para a convecção e sem modelo de solo-superfície urbana.

As configurações avaliadas neste trabalho envolvem três opções no modelo: CLP; Camada Limite Superficial (CLS) e Modelo de Solo-superfície (MS). A escolha do esquema da CLP interfere diretamente na da CLS e na do MS. Cada modelo físico está brevemente documentado no manual do WRF (SKAMAROCK et al., 2008). As escolhas foram divididas em seis experimentos, organizados de acordo com a Tab. 1.

Tabela 1. Experimentos de avaliação dos esquemas físicos de CLP, CLS e de MS.

\begin{tabular}{cccc}
\hline Experimento & CLP & CLS & MS \\
\hline 1 & YSU & MM5 & Noah \\
2 & MYJ & ETA & Noah
\end{tabular}


3

4

5

6
ACM2

QNSE

MYNN2

BouLac
PX

PX

QNSE

Noah

MYNN

Noah

ETA

Noah

Para se comparar os resultados das simulações com as radiossondagens, foram utilizados a Raiz do Erro Quadrático Médio (RMSE) e o Índice de Concordância de Willmott (IA) para alturas de até 5 km (LEGATES; MCCABE, 1999).

\section{RESULTADOS E DISCUSSÃO}

A Tabela 2 mostra os valores de RMSE para cada um dos experimentos. É possível observar que todos apresentaram valores muito próximos entre si, com destaque para o Exp. 3, que apresentou os menores erros.

A Tabela 3 mostra o IA para os experimentos. Observa-se que todos também apresentaram valores muito próximos entre si, satisfatórios, com destaque para os Exp. 2 (velocidade) e 6 (direção), com valores mais próximos de 1 .

Tabela 2. Valores da RMSE para a velocidade e direção do vento em cada um dos experimentos.

\begin{tabular}{lcccccc}
\hline \multicolumn{1}{c}{ Grandeza } & $\mathbf{1}$ & $\mathbf{2}$ & $\mathbf{3}$ & $\mathbf{4}$ & $\mathbf{5}$ & $\mathbf{6}$ \\
\hline Vel. (m/s) & 4,327 & 4,262 & $\mathbf{4 , 1 9 3}$ & 4,391 & 4,276 & 4,278 \\
Direção (graus) & 57,70 & 51,76 & $\mathbf{4 4 , 3 3}$ & 57,62 & 47,65 & 50,39 \\
\hline
\end{tabular}

Tabela 3. Valores do IA para a velocidade e direção do vento em cada um dos experimentos.

\begin{tabular}{|c|c|c|c|c|c|c|}
\hline Grandeza & 1 & 2 & 3 & 4 & 5 & 6 \\
\hline Vel. $(\mathrm{m} / \mathrm{s})$ & 0,580 & 0,614 & 0,597 & 0,555 & 0,607 & 0,608 \\
\hline Direção (graus) & 0,741 & 0,769 & 0,782 & 0,761 & 0,774 & $\mathbf{0 , 8 1 0}$ \\
\hline
\end{tabular}

De uma maneira geral, o WRF apresentou uma tendência de superestimar os valores de velocidade do vento em média $23,5 \%$, principalmente em níveis próximos à superfície e em condições que houve precipitação, o que pode ter causado os altos valores de RMSE. 


\section{CONCLUSÕES}

Os valores de RMSE e IA foram muitos próximos entre si, o que impede de se afirmar que há um único esquema físico que se destaca em relação aos outros. Apesar disto, o IA mostra que o modelo representou de forma satisfatória a velocidade e principalmente a direção do vento para o período.

O WRF apresentou uma tendência geral de superestimar em média $23,5 \%$ a velocidade do vento, principalmente em níveis próximos à superfície e em condições que houve precipitação.

\section{REFERÊNCIAS}

LEGATES, D. R.; MCCABE, G. J. Evaluating the use of "goodness-of-fit" Measures in hydrologic and hydroclimatic model validation. Water Resources Research, v. 35, n. 1, p. 233-241, 1999.

SKAMAROCK, W. C., KLEMP, J. B., DUDHIA, J., GILL, D. O., BARKER, D. M., DUDA, M. G., HUANG, X. Y., WANG, W., POWERS, J. G. A Description of the Advanced Research WRF Version 3: NCAR technical note, NCAR/TN-475+STR, 2008, $126 \mathrm{p}$. 